\title{
Robust Operation of Mesoporous Antireflective Coatings under Variable Ambient Conditions
}

\author{
Barry Reid, ${ }^{\dagger}$ Alaric Taylor, ${ }^{\dagger}$ Yinong Chen, $^{\dagger}$ Benjamin Schmidt-Hansberg, ${ }^{\ddagger}$ and Stefan Guldin ${ }^{\dagger}{ }^{\dagger}$ (0) \\ ${ }^{\dagger}$ Department of Chemical Engineering, University College London, Torrington Place, London WC1E 7JE, U.K. \\ ${ }^{\ddagger}$ Chemical \& Process Engineering, Coating \& Film Processing, BASF SE, Carl-Bosch-Strasse 38, Ludwigshafen am Rhein 67056, \\ Germany
}

Supporting Information

ABSTRACT: Generating mesoporous films with adequate film thickness and refractive index is a common method to achieve amplitude and phase matching in low-cost interference-based antireflective coatings (ARCs). For high-surface-energy materials, pores on the $2-50 \mathrm{~nm}$ (i.e., on the subwavelength scale) are subject to capillary condensation by surrounding gas phase water molecules, which hampers their functioning. In this work, we examine the effect of relative humidity on mesoporous ARCs and present a simple method for the preparation of ARCs with robust operation under variable conditions. The materials route is based on the generation of well-defined porous aluminosilicate networks by block copolymer co-assembly with poly(isobutylene)-block-poly(ethylene oxide) and postsynthesis grafting of trichloro(octyl)silane molecules to the pore walls. The functionalized films exhibited a maximum transmittance value of $99.8 \%$, with an average transmittance of $99.1 \%$ in the visible wavelength range from 400 to $700 \mathrm{~nm}$. Crucially, the antireflection performance was maintained at high humidity values, with an average transmittance decrease of only $0.2 \%$ and maximum values maintained at $99.7 \%$. This compared to maximum and average losses of 3.6 and $2.7 \%$, respectively, for nonfunctionalized reference samples. The ARCs were shown to retain their optical properties within 50 humidity cycles, indicating long-term stability against fluctuating environmental conditions.

KEYWORDS: antireflective coating, optical coating, antireflection, block copolymer, self-assembled monolayer, ellipsometric porosimetry, capillary condensation

\section{INTRODUCTION}

Antireflective coatings (ARCs) are a common component in fields such as eye glasses, display technology, solar cells, and windows. ${ }^{1}$ Light reflection at optical interfaces is a consequence of a contrast in the refractive index, and thus one of the most appealing strategies for ARCs involves the generation of gradient structures on the wavelength scale. ${ }^{2}$ Examples include biomimetic motheye-type features, which consist of subwavelength nanopillars deposited or etched directly on a surface. ${ }^{3}$ Top-down lithographical methods, such as deep-UV, interference or electron beam lithography, were first utilized to replicate these structures. ${ }^{4}$ Modern bottom-up methods for forming lithographic masks employ self-assembled polystyrene colloids, ${ }^{5}$ metallic nanoparticles, ${ }^{6}$ or block copolymers. ${ }^{7}$ However, these methods are typically challenged by convoluted processes with numerous fabrication steps and issues related to their scalability. ${ }^{3}$

Interference-based ARCs offer an alternative approach to the above methods. A single-layer coating must fulfill two requirements: ${ }^{8}(1)$ the optical path length of the film $n_{\text {film }} \times$ $d$ must be one quarter of the wavelength of the incident light $(\lambda / 4)$ and (2) the refractive index (RI) of the material needs to equate to the square root of the substrate RI
$\left(n_{\mathrm{ARC}}=\sqrt{n_{\text {air }} n_{\text {substrate }}}\right)$. Given that ARCs are typically coated on glass substrates with RI of around 1.5, a thin film fulfilling antireflection (AR) conditions should have a target RI value $\approx 1.22-1.23$. Dense solids do not exhibit an RI value below 1.3, and thus to achieve interference-based $A R$ in a single layer, the introduction of porosity on the subwavelength scale is required.

Spin-coated polymer blends produced sub-nanometer structures via selective solvent etching of one component. ${ }^{9}$ Other deposition methods include convective self-assembly ${ }^{10}$ and electrostatic deposition ${ }^{11}$ of silica nanoparticle-based materials. Preformed silica nanoparticles have been combined with sol-gel-based silica "binder" to obtain ARCs, albeit at higher than optimum RI values. ${ }^{12}$ Recently, coatings with AR properties were produced from a sequential infiltration method that introduced gas phase alumina into swollen polymer films using atomic layer deposition. ${ }^{13}$ Broadband functioning is facilitated by multilayer assemblies. ${ }^{14}$ Structure-directing agents such as surfactants and block copolymers (BCPs) have been

Received: December 1, 2017

Accepted: February 14, 2018

Published: February 14, 2018 
extensively studied for the formation of a variety of inorganic mesoporous thin film architectures with closely controlled porosity and pore size. ${ }^{15-17}$ Well-established sol-gel chemistry methods, when combined with solution-based BCPs, allow for the facile preparation of mesoporous ARCs via conventional liquid deposition processes. ${ }^{18-21}$ Incorporation of $\mathrm{TiO}_{2}$ into mesoporous ARCs allows for the degradation of organic contaminants via photocatalysis. This has been achieved via integration of preformed nanocrystals, ${ }^{20}$ ultrathin crystalline, ${ }^{22}$ or mesoporous titania layers. ${ }^{23}$

A practical problem of mesoporous ARCs, in particular, when made of high-surface-energy inorganic materials, is that pores on the subwavelength scale are susceptible to filling with ambient gas phase water molecules via capillary condensation, ${ }^{24}$ thus resulting in a reduction of the AR effect. Hydrophobicity to suppress capillary condensation may be conferred by incorporating nonpolar functional groups during synthesis via co-condensation methods or by postsynthetic grafting. ${ }^{25,26} \mathrm{Co}-$ condensation methods generally combine conventional silica with hydrophobic silane precursors. ${ }^{19,27-30}$

Experimental procedures are hampered by extensive sol aging times, often on the scale of days or weeks. ${ }^{1,27-29}$ Furthermore, consideration must be given to the role and compatibility of the organic component and how it influences the hydrolysis and condensation processes. ${ }^{31}$ The potential loss of organic functionality during high-temperature template removal ${ }^{32}$ and limited exposure of functional groups within the material backbone ${ }^{33}$ pose further challenges.

Although postsynthetic grafting removes uncertainty regarding precursor incompatibility and control over the sol-gel process and, furthermore, is more efficient in increasing the hydrophobicity, ${ }^{26,34}$ the approach suffers from other shortfalls. Grafting inherently results in porosity reduction and an increase in the refractive index. Other issues arise from possible pore blocking and limitations regarding diffusion of the functional material into the pores. ${ }^{31}$ Substrates are typically submerged in solutions ${ }^{18,35}$ or exposed to vapors ${ }^{19,36}$ of the grafting agent for a period of time. Recently, a number of studies were reported on the successful generation of grafted ARCs. ${ }^{18,27,35,37}$ However, previous reports lacked in situ characterization of the optical properties as a function of humidity. Furthermore, the porosity values and thus refractive indices were not matched to obtain ideal AR performance after grafting.

In this work, we describe a simple, low-cost preparation of mesoporous aluminosilicate materials using the amphiphilic poly(isobutylene)-block-poly(ethylene oxide) (PIB- $b$-PEO) block copolymer as a structure-directing agent mixed with aluminosilicate material derived from sol-gel chemistry. This approach allows systematic tuning of the porosity value of the ARC to ensure ideal amplitude and phase matching after grafting of trichloro(octyl)silane molecules to the pore walls. The optical properties of the resulting ARCs are studied in repeated cycles under variable humidity by ellipsometric porosimetry (EP) and in situ optical transmittance measurements, and conclusions are drawn for the robust operation of mesoporous ARCs under variable ambient conditions.

\section{EXPERIMENTAL SECTION}

2.1. Preparation of Mesoporous Antireflective Coating. $\mathrm{PIB}_{39}-b-\mathrm{PEO}_{36}$ block copolymer was synthesized by BASF with $M_{\mathrm{n}}$ of $4.85 \mathrm{~kg} / \mathrm{mol}$ and polydispersity of 1.26 , as described elsewhere. ${ }^{38}$ The BCP was dissolved in an azeotrope mixture of toluene (99.9\%, Sigma-Aldrich) and 1-butanol (99.4\%, Sigma-Aldrich); concentrations are mentioned below. Aluminosilicate sol was prepared as described in detail elsewhere. ${ }^{20}$ Briefly, $2.8 \mathrm{~g}$ of (3-glycidyloxypropyl)trimethoxysilane ( $\geq 98 \%$, Sigma-Aldrich) was mixed with $0.32 \mathrm{~g}$ of aluminum tri-sec-butoxide (97\%, Sigma-Aldrich) (reactant mole ratio of 9:1) and $20 \mathrm{mg}$ of $\mathrm{KCl}(\geq 99.9 \%$, Sigma-Aldrich). The solution was placed in an ice bath and stirred vigorously for $15 \mathrm{~min}$. The initial hydrolysis step involved the slow, dropwise addition of $0.135 \mathrm{~mL}$ of 10 $\mathrm{mM} \mathrm{HCl}$ in water. The solution was stirred for $15 \mathrm{~min}$ at $0{ }^{\circ} \mathrm{C}$, followed by a further $15 \mathrm{~min}$ of stirring at room temperature. For complete hydrolysis, $0.85 \mathrm{~mL}$ of $10 \mathrm{mM} \mathrm{HCl}$ in water was added and the mixture was stirred for a further $20 \mathrm{~min}$. The final sol was then filtered using a $0.2 \mu \mathrm{m}$ syringe filter before addition to the polymer solution in the desired inorganic-to-organic (I/O) ratio. The as-made sol was then diluted with the azeotrope solvent mixture to a concentration of $1000 \mathrm{mg} / \mathrm{mL}$ and stored at $5{ }^{\circ} \mathrm{C}$ for further use, with a shelf life of at least one month. The high-porosity samples for silane functionalization were prepared from hybrid solutions consisting of an $\mathrm{I} / \mathrm{O}$ ratio of $1: 1$ (denoted as $\mathrm{ARC}_{1-1 n f}$ and $\mathrm{ARC}_{1: 1 f}$ for nonfunctionalized and functionalized versions, respectively). PIB- $b$-PEO $(50 \mathrm{mg}$ ) was dissolved in $1.12 \mathrm{~mL}$ of azeotrope solution, followed by the addition of aluminosilicate sol material $(100 \mathrm{mg}$, corresponding to approximately $50 \mathrm{mg}$ of aluminosilica). ${ }^{39}$ For the reference ARCs without functionalization, hybrid solutions with an I/O ratio of 3:1 $\left(\mathrm{ARC}_{3: 1}\right)$ were prepared to match the RI requirements. For these samples, $50 \mathrm{mg}$ of polymer was dissolved in $1.724 \mathrm{~mL}$ of azeotrope solvent, followed by addition of $300 \mathrm{mg}$ of aluminosilica sol. The hybrid solutions were then placed on a shaker at $600 \mathrm{rpm}$ for $1 \mathrm{~h}$ before deposition onto substrates. To produce thin films, the solutions were spin-coated on silicon (University Wafer, single-side polished, $640 \mu \mathrm{m}$ thick, p-doped, $20-24 \Omega \mathrm{cm}$ ) and glass (Pilkington Microwhite) substrates $(1500 \mathrm{rpm}, 20 \mathrm{~s})$. All substrates were first treated with oxygen plasma for $5 \mathrm{~min}$, followed by cleaning with a Snowjet $\mathrm{CO}_{2}$ cleaner (Megatech Ltd.) immediately before deposition. The as-made hybrid films were annealed on a hot plate to a temperature of $130{ }^{\circ} \mathrm{C}\left(1{ }^{\circ} \mathrm{C} / \mathrm{min}\right.$ ramp, $30 \mathrm{~min}$ dwell time $)$, followed by calcination in a furnace at $4500^{\circ} \mathrm{C}$ for $1 \mathrm{~h}$.

2.2. Hydrophobic Functionalization. Samples were allowed to cool post calcination and subsequently placed in a covered glass Petri dish containing trichloro(octyl)silane (TOS, 97\%, Sigma-Aldrich) diluted to $0.1 \mathrm{mM}$ in heptane ( $\geq 99.9 \%$, Sigma-Aldrich) for $5 \mathrm{~h}$. Following removal, all samples were washed thoroughly using heptane and dried under a stream of nitrogen.

2.3. Material Characterization. Spectroscopic ellipsometry (SE) and environmental water-based ellipsometric porosimetry (EP) measurements were performed using a spectroscopic ellipsometer (Semilab SE2000) and analyzed with the software of the manufacturer (SEA). EP measurements with toluene as sorbent were performed under vacuum in a dedicated chamber using a Semilab PS2000 ellipsometer. All measurements were carried out at an incident angle of $73^{\circ}$ on a silicon substrate. Data fitting was performed using a standard Cauchy dispersion law. The Lorentz-Lorentz effective medium approximation model was used to calculate the relative volume of adsorbed water (or toluene) on the basis of the polarizability and molar volume of the sorbent. ${ }^{40}$ Prior to EP measurements, samples were heated on a hot plate at $120^{\circ} \mathrm{C}$ for several minutes to ensure the exclusion of residual water in the pores. Transmittance measurements were performed using a Zeiss Axioscope Al with $5 \times$ magnification coupled with $400 \mu \mathrm{m}$ optical fiber (Ocean Optics) and a highresolution spectrometer (Ocean Optics, QE65000). Humidity was controlled during in situ transmittance measurements using mass flow controllers (Bronkhorst, F-201CV-100) connected to an enclosed microscope stage (Linkam, LTS120), which was modified with the integration of a humidity sensor. All relative humidity $(\mathrm{RH})$ values were reported with an accuracy of $\pm 5 \%$. Measurements were recorded $1 \mathrm{~h}$ after stabilization of the desired relative humidity value. Contact angle measurements were performed on a drop-shaped analyzer (Kruss, DSA100). Scanning electron microscopy (SEM) images were carried out on a Carl Zeiss Orion Nanofab at an accelerating voltage of $25 \mathrm{kV}$. Samples were sputter-coated with platinum before imaging. To probe the mesostructure in the bulk rather than on the surface of the 


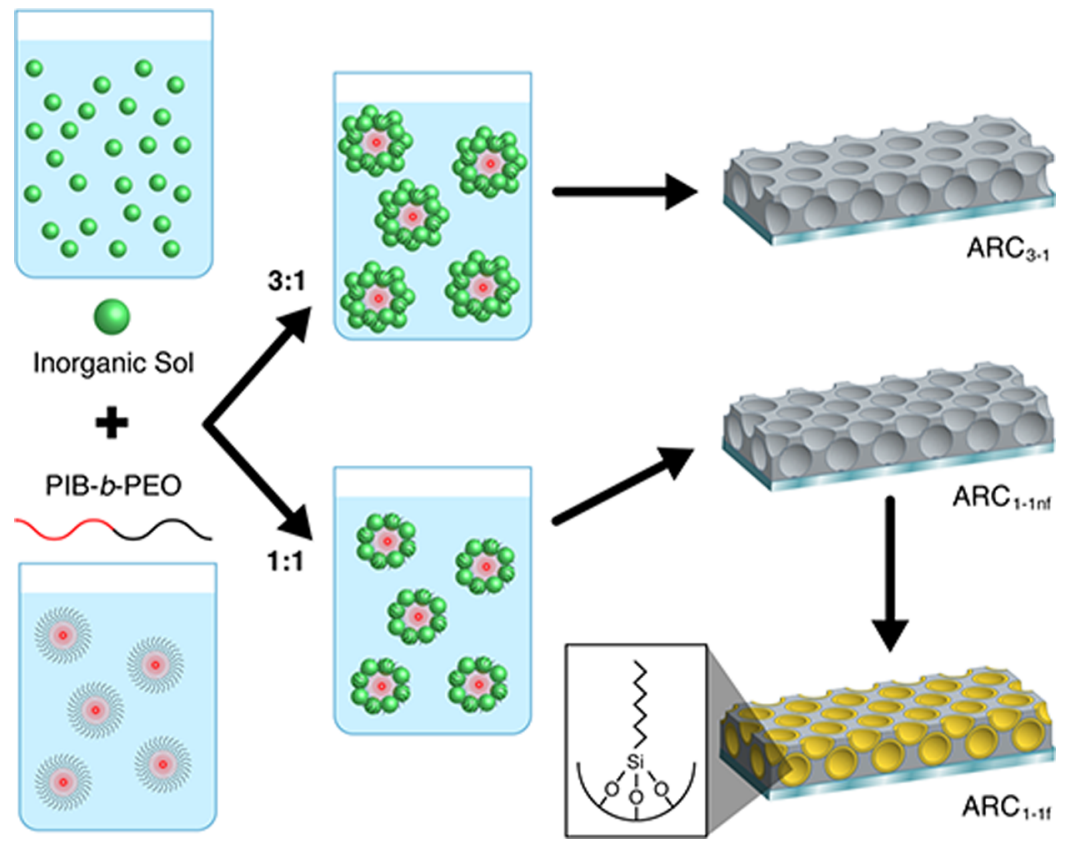

Figure 1. Schematic representation of the ARC preparation process.

coating, reactive ion etching was carried out on the annealed hybrid samples before removal of the organic compounds (Oxford Instruments, PlasmaPro NGP80). The process was conducted with a $200 \mathrm{~W}$ direct current bias whilst the chamber (cleaned and preconditioned) was held at 30 mTorr with a continuous flow of $12 \mathrm{sccm} \mathrm{CHF}_{3}$ and 38 sccm Ar. Atomic force microscopy was performed using a Bruker Dimension Icon and Bruker SAA-HPI-SS probe (nominal tip radius 1 $\mathrm{nm}$ ). The scans were performed in PeakForce mode at a scan rate of $400 \mathrm{~nm} / \mathrm{s}$, with a peak force amplitude of $30 \mathrm{~nm}$ and frequency of 2 $\mathrm{kHz}$.

\section{RESULTS AND DISCUSSION}

A schematic of the synthetic approach is shown in Figure 1. Two sets of samples were prepared with different porosity values based on their $\mathrm{I} / \mathrm{O}$ ratio. SE and EP measurements allowed the accurate and reproducible measurement of the refractive index, thickness, and porosity (Table S1, Supporting Information). $\mathrm{ARC}_{3: 1}$ samples exhibited an average $\mathrm{RI}$ of $1.22 \pm$ 0.02 and a porosity of $49 \pm 4 \%$, thus being ideal reference samples for nonfunctionalized ARCs.

SEM and EP measurements (Figure 2a,b) confirm the presence of a continuous porous network with an RI value of $\approx 1.22$ in dry atmosphere. The corresponding volume-adsorbed isotherm (Figure $2 \mathrm{c}$ ) affirms a porosity of $\approx 50 \%$. EP provides further information on the porous network on the basis of the isotherm characteristics. For $\mathrm{ARC}_{3: 1}$, the isotherm shape was found to be consistent with a type IV IUPAC classification associated to mesoporous materials. ${ }^{41}$ The broadness of the loop and gradual slope of the adsorption curve is in line with a type $\mathrm{H} 2$ hysteresis attributed to "ink bottle" type pore interconnections. In this geometry, which is consistent with the observed inverse opal-type morphology, the emptying of nonsurface pores during desorption is limited by the dimensions of the pore necks. ${ }^{42,43}$ These observations are in line with results obtained by atomic force microscopy, see the Supporting Information (Figure S1). On the basis of Figure 2b, it is evident that $\mathrm{ARC}_{3: 1}$ samples undergo capillary condensation in the range of $45-60 \%$ relative humidity $(\mathrm{RH})$, thus no longer fulfilling the requirements for $\mathrm{AR}$. (a)
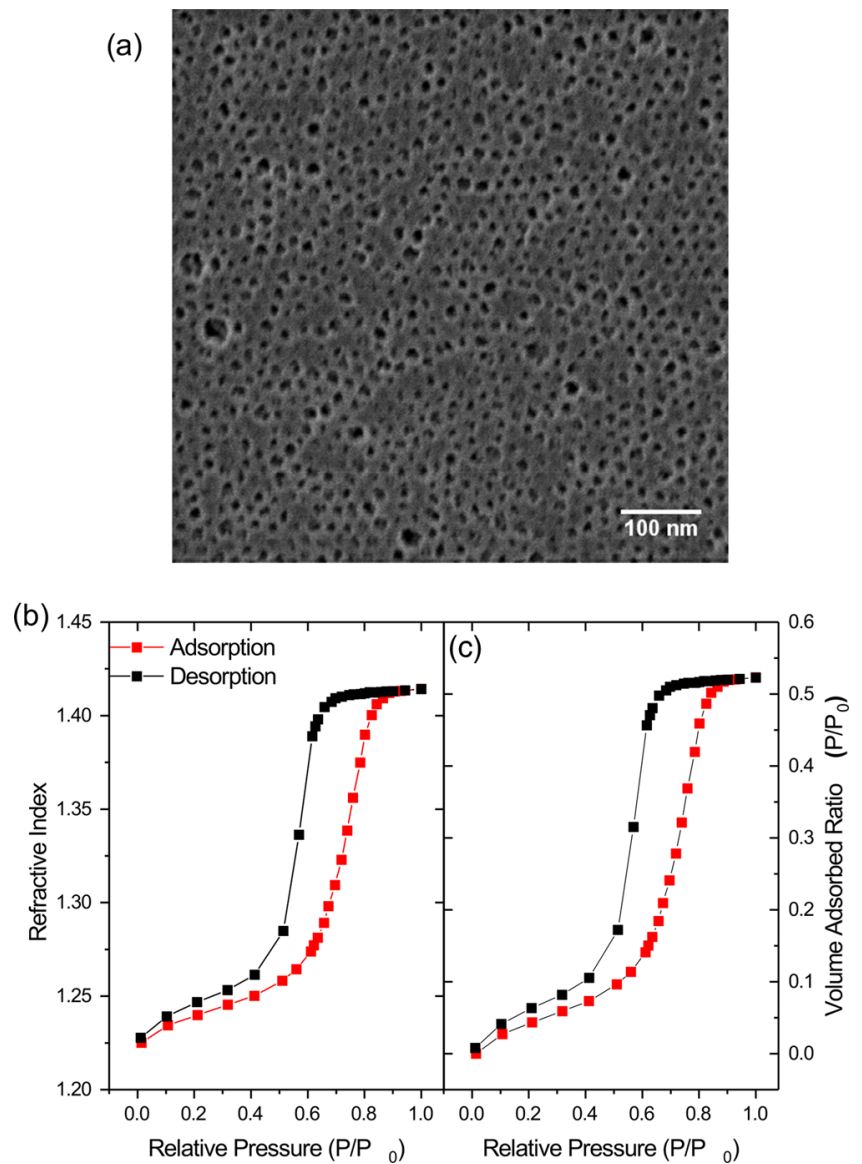

Figure 2. Nonfunctionalized $\mathrm{ARC}_{3: 1}$ sample: top-view SEM image (a), refractive index isotherm measured by environmental ellipsometric porosimetry with water as sorbent, (b) and the corresponding volumeadsorbed isotherm (c).

Decreasing the $\mathrm{I} / \mathrm{O}$ ratio in the formulation to $1: 1$ led to $\mathrm{ARC}_{1: 1 n f}$ samples with a mean RI value of $1.12 \pm 0.01$ and a porosity of $70 \pm 2 \%$. These results demonstrate the versatility 
of block copolymers for the preparation of porous materials with target and extremely RI values. Further structural characterization can be found in the Supporting Information (Figure S2). The structure formation principle via dense packing of sol-loaded block copolymer micelles allows tuning the RI over a wide range by adjusting the $\mathrm{I} / \mathrm{O}$ ratio. ${ }^{15}$ As shown in the Supporting Information (Figure S3), the measured porosity and RI values compare favorably with similar samples that were prepared from higher molecular weight poly(isoprene)-block-poly(ethylene oxide) (PI-b-PEO) block copolymers. $^{20}$ These low RI values provide the potential for incorporating functional materials into the porous network while still maintaining $\mathrm{AR}$ conditions. Here, $\mathrm{ARC}_{1: 1 n f}$ samples were placed in a solution of trichloro(octyl)silane (TOS, RI $\approx$ $1.45^{44}$ ) to functionalize the surface with nonpolar long-chain hydrocarbons via the reaction of the chlorosilane with exposed hydroxyl groups on the surface of the porous network (see also Figure 1). Trichlorosilanes were chosen as they exhibited greater surface coverage on silica materials when compared with monochloro or dichloro moieties. ${ }^{45}$

Figure 3 shows the effect of silane functionalization on the characteristics of the $\mathrm{ARC}_{1: 1}$ samples. The water contact angle

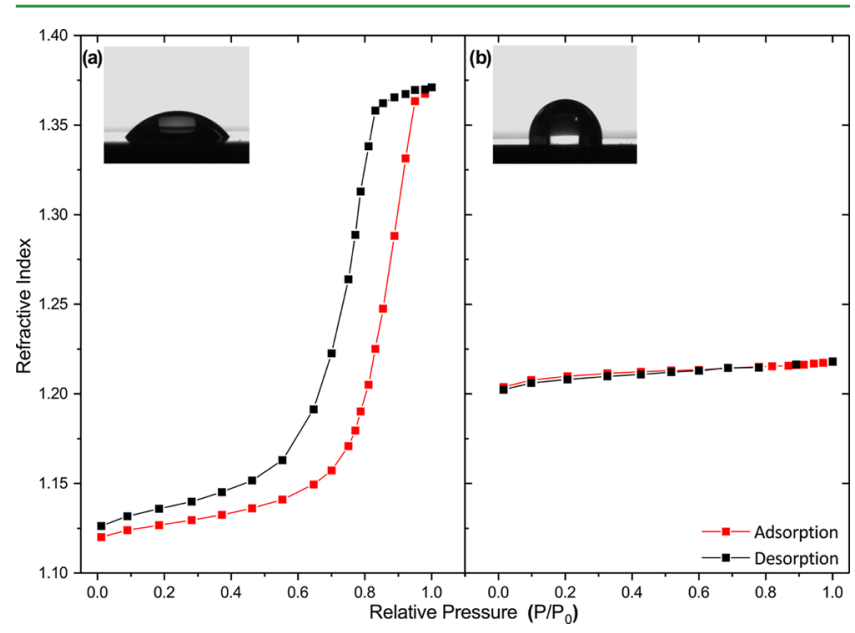

Figure 3. $\mathrm{EP}$ isotherms of $\mathrm{ARC}_{1: 1}$ samples before (a) and after (b) functionalization with TOS. The insets depict the corresponding macroscopic contact angle measurements.

(Figure 3, inset) increased from $52 \pm 2^{\circ}$ in $\mathrm{ARC}_{1: 1 n f}$ to $99 \pm 2^{\circ}$ in $\mathrm{ARC}_{1: 1 f}$, demonstrating an increase in hydrophobicity in general terms. Macroscopic contact angle measurements, however, do not provide direct information on the hydrophobicity of the pores. ${ }^{36}$

Although EP is established as a powerful technique for the characterization of mesoporous thin films, here we demonstrate its value in characterizing mesopore wetting properties. Prior to TOS functionalization (Figure 3a), the onset of capillary condensation was observed at $\mathrm{RH} \approx 75 \%$ in $\mathrm{ARC}_{1: 1 n f}$, whereas all pores were filled with water at $\mathrm{RH} \approx 95 \%$. The $\mathrm{ARC}_{1: 1 n f}$ isotherm exhibited shape and hysteresis characteristics similar to those observed in the $\mathrm{ARC}_{3: 1}$ sample (Figure 2a). For the hydrophobic $\mathrm{ARC}_{1: 1 \mathrm{f}}$, water-based $\mathrm{EP}$ measurements (Figure $3 \mathrm{~b})$ presented evidence that the functionalized pores were capable of preventing significant water adsorption, even under extreme humidity conditions. In this instance, a small increase in RI was determined at high humidity, equivalent to $4.7 \%$ uptake of water into the pores, which compared to $\approx 70 \%$ in $\mathrm{ARC}_{1: 1 n f}$. Crucially, no capillary condensation was observed, and even at conditions close to saturation, the optimum RI value of 1.22 was not exceeded. For further characterization of the hydrophobic mesoporous network in $\mathrm{ARC}_{1: 1 \mathrm{f}}$, toluenebased EP was carried out in a dedicated chamber. This approach allowed to probe the accessibility of the porous network with a nonpolar sorbent. A porosity value of $\approx 49.1 \%$ was measured using this technique (Figure S4). These results are in line with a volume estimation of TOS within the $\mathrm{ARC}_{1: 1 f}$ network by a Bruggeman effective medium approximation (eq 1 , Supporting Information). On the basis of values obtained via EP and spectroscopic ellipsometry, a volume fraction of $\approx 20.6 \%$ of TOS was calculated, whereas porosity was estimated to be $\approx 51.1 \%$. These calculations are broadly in agreement with the experimental toluene-based EP results. See the Supporting Information for further details.

EP was also used to monitor the stability of the functionalized coatings when subjected to repeated dry and wet air cycles. This approach provides a viable alternative for the testing of environmental durability of coatings, compared to the standard tests that involve exposing the sample to constant $\mathrm{RH}$ values for extended periods of time followed by measurement of the material properties ex situ. ${ }^{19,46}$ In earlier works on silica-based networks, repeated cycles of water adsorption and desorption demonstrated an impact on the mechanical integrity of the porous skeleton as well as hydrophobicity via capillary stresses and irreversible adsorption of water molecules within the pores. ${ }^{4-49}$ In the present study, the $\mathrm{ARC}_{1: 1 f}$ sample maintained both its hydrophobicity and $\mathrm{AR}$ capabilities after 50 EP cycles. The average RI at 97\% RH was $\approx 1.22$, with none of the RI measurements exceeding 1.23 (Figure S5). Previous studies determined the effect of adsorption and desorption of water by varying temperature. ${ }^{50}$ To our knowledge, however, this is the first reported work on the effects of humidity cycling on ARC stability.

When light passes through float glass, intensity is lost from reflections at each air/glass interface. Typically, this results in the loss of $\approx 4 \%$ of transmitted light at each interface. In the present study, uncoated Pilkington Microwhite exhibited an average transmittance of $92.4 \%$ between wavelengths of $400-$ $700 \mathrm{~nm}$, with a maximum value of $92.6 \%$. When $\mathrm{ARC}_{3: 1}$ was coated on both sides, this increased to an average transmittance of $98.8 \%$, with a maximum of $99.9 \%$ at $454 \mathrm{~nm}$. The functionalized $\mathrm{ARC}_{1: 1 f}$ coating exhibited a slightly higher average transmittance of $99.1 \%$, with a maximum transmittance of $99.8 \%$ at a wavelength of $461 \mathrm{~nm}$.

To demonstrate the functioning of the AR coatings under variable environmental conditions, samples were placed in a humidity-controlled chamber and in situ transmittance measurements were recorded. When $\mathrm{ARC}_{3: 1}$ samples were exposed to an atmosphere with $\mathrm{RH} \approx 97 \%$ (Figure $4 \mathrm{a}$ ), the transmittance almost instantly decreased from an average value of 98.8 to $96.1 \%$, corresponding to a reduction in mean transmittance by $2.7 \%$, with a maximum change of $-3.6 \%$ (Figure 4c). These results are in line with data obtained from EP (Figure 2), demonstrating the complete filling of pores at this $\mathrm{RH}$ level.

In contrast to the $\mathrm{ARC}_{3: 1}$ sample, the mean transmittance of the functionalized $\mathrm{ARC}_{1: 1 f}$ samples exhibited only a small decrease of $0.2 \%$ to $98.9 \%$, with the maximum value remaining at $99.7 \%$ when exposed to the same level of humidity. Comparing changes in both samples at high humidity (Figure $4 \mathrm{c})$, the $\mathrm{ARC}_{1: 1 f}$ maintained an average improvement of $\approx 2.7 \%$ over $\mathrm{ARC}_{3: 1}$, with a maximum transmittance difference of 

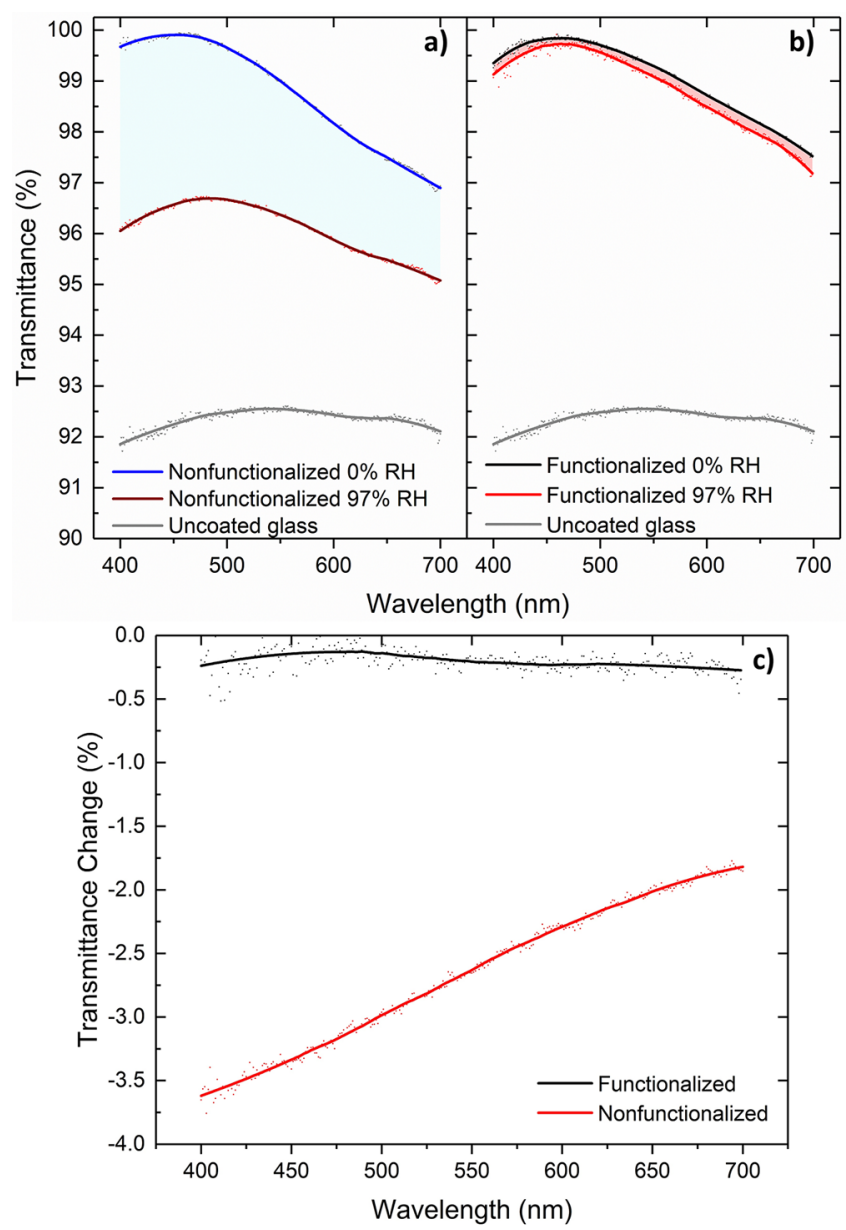

Figure 4. Optical transmittance vs relative humidity. (a) Nonfunctionalized sample $\mathrm{ARC}_{3: 1}$, (b) functionalized $\mathrm{ARC}_{1: 1 f}$ sample, and (c) net transmittance change at $97 \%$ relative humidity.

$\approx 3.1 \%$. The results described herein compare favorably with previous reports on hydrophobic ARCs synthesized via grafting $^{18,35,37}$ and co-condensation methods. ${ }^{28,30,46}$ In contrast to earlier reports, these results were based on in situ EP and transmittance measurements, thereby providing evidence for the effectiveness of the $\mathrm{ARC}_{1: 1 f}$ samples in withstanding humidity conditions near saturation and repeated humidity cycles. Although this study proves the stability of the silane monolayer under humid conditions, we did not conduct further investigations into the long-term effect of exposure to sunlight. Alkane monolayers are susceptible to photo-oxidation when exposed to UV radiation and oxygen. ${ }^{51}$ In comparison to thiol bonds, the siloxane bond itself is less sensitive to photolysis and thus degradation is more likely to occur along the alkyl chain. ${ }^{52}$ Our work is compatible with a broad library of silane-based ligands, and the molecular design choice for long-term stability will be further investigated in a subsequent study. Finally, we note that our approach also presents the possibility of grafting trichlorosilyl derivatives with alternative functionality within the aluminosilicate mesopores, with the aim of developing mesoporous thin films for other applications. ${ }^{53,54}$

\section{CONCLUSIONS}

In conclusion, we report in this work on materials and characterization aspects of mesoporous ARCs for robust operation under variable ambient conditions. Ellipsometric porosimetry and transmission experiments with controlled relative humidity enabled to study in situ the impact of humidity-induced capillary condensation on the optical properties of ARCs. The use of an amphiphilic PIB- $b$-PEO block copolymer as a structure-directing agent for the co-assembly of aluminosilicate material derived from sol-gel chemistry allowed to deposit mesoporous optical coatings with broad porosity and thus perform refractive index tuning. ARCs were fabricated to meet amplitude- and phase-matching conditions after grafting of a hydrophobic agent to the pore walls, in this case trichloro(octyl)silane (TOS). As a result, near-optimum optical performance was achieved under variable atmospheric conditions, with a maximum transmittance value for doublesided substrates of $99.8 \%$ under dry and $99.7 \%$ near saturation, and an average transmittance in the wavelength range from 400 to $700 \mathrm{~nm}$ of 99.1 and $98.9 \%$, respectively. The ARCs were shown to withstand repeated humidity cycles and offer a reliable route to low-cost solution-processable coatings on glass and other transparent substrates.

\section{ASSOCIATED CONTENT}

\section{Supporting Information}

The Supporting Information is available free of charge on the ACS Publications website at DOI: 10.1021/acsami.7b18299.

Volume determination of film components by effective medium approximation; overview of measured parameters for ARC samples; $\mathrm{AFM}$ scan of $\mathrm{ARC}_{1: 1}$; top-view SEM image and $\mathrm{AFM}$ scan of $\mathrm{ARC}_{3: 1}$ sample; refractive index and corresponding porosity of optical coatings as a function of polymer weight content; EP isotherms of $\mathrm{ARC}_{1: 1 f}$ using toluene as sorbent; refractive index values of $\mathrm{ARC}_{1: 1 f}$ samples during multiple EP cycles (PDF)

\section{AUTHOR INFORMATION}

\section{Corresponding Author}

*E-mail: s.guldin@ucl.ac.uk.

\section{ORCID}

Stefan Guldin: 0000-0002-4413-5527

\section{Notes}

The authors declare no competing financial interest.

\section{ACKNOWLEDGMENTS}

B.R. acknowledges funding by an EPSRC Industrial Case Award (EP/M506448/1) in support of BASF SE. A.T. recognizes support by the EPSRC Doctoral Training Partnership scheme (EP/M507970/1). S.G. is grateful for a start-up fund from the Department of Chemical Engineering, University College London. The authors acknowledge Dr. Peter Basa and Dr. Akos Botos from Semilab (Budapest, Hungary) for carrying out EP measurements with toluene as sorbent. Furthermore, the authors are grateful to Dr. Jan Gebers (BASF Schweiz AG) for useful discussions and Salma Conway for assistance in preparation of schematic images.

\section{REFERENCES}

(1) Yao, L.; He, J. Recent progress in antireflection and self-cleaning technology-From surface engineering to functional surfaces. Prog. Mater. Sci. 2014, 61, 94-143.

(2) Gombert, A.; Glaubitt, W.; Rose, K.; Dreibholz, J.; Bläsi, B.; Heinzel, A.; Sporn, D.; Döll, W.; Wittwer, V. Subwavelengthstructured antireflective surfaces on glass. Thin Solid Films 1999, $351,73-78$. 
(3) Chattopadhyay, S.; Huang, Y. F.; Jen, Y. J.; Ganguly, A.; Chen, K. H.; Chen, L. C. Anti-reflecting and photonic nanostructures. Mater. Sci. Eng., R 2010, 69, 1-35.

(4) Rafaelsen, L.; Christrup, H.; Bech, P.; Rafaelsen, O. J. Reduction of lens reflexion by the "Moth Eye" principle. Nature 1973, 242, 117118.

(5) Kuo, W. K.; Hsu, J. J.; Nien, C. K.; Yu, H. H. Moth-eye-inspired biophotonic surfaces with antireflective and hydrophobic characteristics. ACS Appl. Mater. Interfaces 2016, 8, 32021-32030.

(6) Lohmüller, T.; Helgert, M.; Sundermann, M.; Brunner, R.; Spatz, J. P. Biomimetic interfaces for high-performance optics in the deep-UV light range. Nano Lett. 2008, 8, 1429-1433.

(7) Päivänranta, B.; Sahoo, P. K.; Tocce, E.; Auzelyte, V.; Ekinci, Y.; Solak, H. H.; Liu, C. C.; Stuen, K. O.; Nealey, P. F.; David, C. Nanofabrication of broad-band antireflective surfaces using selfassembly of block copolymers. ACS Nano 2011, 5, 1860-1864.

(8) Rancourt, J. D. Optical Thin Films: User Handbook; SPIE Optical Engineering Press, 1996; p 289.

(9) Walheim, S.; Schäffer, E.; Mlynek, J.; Steiner, U. Nanophaseseparated polymer films as high-performance antireflection coatings. Science 1999, 283, 520-5222.

(10) Prevo, B. G.; Hwang, Y.; Velev, O. D. Convective assembly of antireflective silica coatings with controlled thickness and refractive index. Chem. Mater. 2005, 17, 3642-3651.

(11) Hattori, H. Anti-reflection surface with particle coating deposited by electrostatic attraction. Adv. Mater. 2001, 13, 51-54.

(12) Moghal, J.; Kobler, J.; Sauer, J.; Best, J.; Gardener, M.; Watt, A. A. R.; Wakefield, G. High-performance, single-layer antireflective optical coatings comprising mesoporous silica nanoparticles. ACS Appl. Mater. Interfaces 2012, 4, 854-859.

(13) Berman, D.; Guha, S.; Lee, B.; Elam, J. W.; Darling, S. B.; Shevchenko, E. V. Sequential infiltration synthesis for the design of low refractive index surface coatings with controllable thickness. ACS Nano 2017, 11, 2521-2530.

(14) Raut, H. K.; Ganesh, V. A.; Nair, A. S.; Ramakrishna, S. Antireflective coatings: A critical, in-depth review. Energy Environ. Sci. 2011, 4, 3779.

(15) Guldin, S.; Kolle, M.; Stefik, M.; Langford, R.; Eder, D.; Wiesner, U.; Steiner, U. Tunable mesoporous Bragg reflectors based on block-copolymer self-assembly. Adv. Mater. 2011, 23, 3664-3668.

(16) Stefik, M.; Guldin, S.; Vignolini, U.; Wiesner, U.; Steiner, U. Block copolymer self-assembly for nanophotonics. Chem. Soc. Rev. 2015, 44, 5076-5091.

(17) Lokupitiya, H. N.; Jones, A.; Reid, B.; Guldin, S.; Stefik, M. Ordered mesoporous to macroporous oxides with tunable isomorphic architectures solution criteria for persistent micelle templates. Chem. Mater. 2016, 28, 1653-1667.

(18) Sun, J.; Zhang, C.; Zhang, C.; Ding, R.; Xu, Y. Effect of posttreatment on ordered mesoporous silica antireflective coating. RSC Adv. 2014, 4, 50873-50881.

(19) Sun, J.; Cui, X.; Zhang, C.; Zhang, C.; Ding, R.; Xu, Y. A broadband antireflective coating based on a double-layer system containing mesoporous silica and nanoporous silica. J. Mater. Chem. C 2015, 3, 7187-7194.

(20) Guldin, S.; Kohn, P.; Stefik, M.; Song, J.; Divitini, G.; Ecarla, F.; Ducati, C.; Wiesner, U.; Steiner, U. Self-cleaning antireflective optical coatings. Nano Lett. 2013, 13, 5329-5335.

(21) Bernsmeier, D.; Polte, J.; Ortel, E.; Krahl, T.; Kemnitz, E.; Kraehnert, R. Antireflective coatings with adjustable refractive index and porosity synthesized by micelle-templated deposition of $\mathrm{MgF}_{2}$ sol particles. ACS Appl. Mater. Interfaces 2014, 6, 19559-19565.

(22) Faustini, M.; Nicole, L.; Boissière, C.; Innocenzi, P.; Sanchez, C.; Grosso, D. Hydrophobic, antireflective, self-cleaning, and antifogging sol-gel coatings: An example of multifunctional nanostructured materials for photovoltaic cells. Chem. Mater. 2010, 22, 4406-4413.

(23) Jin, B.; He, J.; Yao, L.; Zhang, Y.; Li, J. Rational design and construction of well-organized macro-mesoporous $\mathrm{SiO}_{2} / \mathrm{TiO}_{2}$ nano- structure toward robust high-performance self-cleaning antireflective thin films. ACS Appl. Mater. Interfaces 2017, 9, 17466-17475.

(24) Horikawa, T.; Do, D. D.; Nicholson, D. Capillary condensation of adsorbates in porous materials. Adv. Colloid Interface Sci. 2011, 169, $40-58$.

(25) Hoffmann, F.; Cornelius, M.; Morell, J.; Fröba, M. Silica-based mesoporous organic-inorganic hybrid materials. Angew. Chem., Int. Ed. 2006, 45, 3216-3251.

(26) Nicole, L.; Boissière, C.; Grosso, D.; Quach, A.; Sanchez, C. Mesostructured hybrid organic-inorganic thin films. J. Mater. Chem. 2005, 15, 3598.

(27) Cai, S.; Zhang, Y.; Zhang, H.; Yan, H.; Lv, H.; Jiang, B. Sol-gel preparation of hydrophobic silica antireflective coatings with low refractive index by base/acid two-step catalysis. ACS Appl. Mater. Interfaces 2014, 6, 11470-11475.

(28) Xia, B.; Luo, J.; Li, Y.; Yang, B.; Zhang, S.; Jiang, B. Preparation of sponge-like porous $\mathrm{SiO}_{2}$ antireflective coatings with excellent environment-resistance by an acid-catalysed sol-gel method. RSC Adv. 2017, 7, 26834-26838.

(29) Cui, X.; Ding, R.; Wang, M.; Wang, C.; Zhang, J.; Wang, J.; Dong, W.; Xu, Y. A hydrophobic and abrasion-resistant $\mathrm{MgF}_{2}$ coating with an ultralow refractive index for double-layer broadband antireflective coatings. J. Mater. Chem. C 2017, 5, 3088-3096.

(30) Li, T.; He, J. Mechanically robust, humidity-resistant, thermally stable high performance antireflective thin films with reinforcing silicon phosphate centers. Sol. Energy Mater. Sol. Cells 2017, 170, 95101.

(31) Sanchez, C.; Boissière, C.; Grosso, D.; Laberty, C.; Nicole, L. Design, synthesis, and properties of inorganic and hybrid thin films having periodically organized nanoporosity. Chem. Mater. 2008, 20, 682-737.

(32) Matheron, M.; Bourgeois, A.; Brunet-Bruneau, A.; Albouy, P.-A.; Biteau, J.; Gacoin, T.; Boilot, J.-P. Highly ordered CTAB-templated organosilicate films. J. Mater. Chem. 2005, 15, 4741.

(33) Calvo, A.; Joselevich, M.; Soler-Illia, G. J.; Williams, F. J. Chemical reactivity of amino-functionalized mesoporous silica thin films obtained by co-condensation and post-grafting routes. Microporous Mesoporous Mater. 2009, 121, 67-72.

(34) Matheron, M.; Bourgeois, A.; Gacoin, T.; Brunet-Bruneau, A.; Albouy, P.-A.; Boilot, J.-P.; Biteau, J.; Lacan, P. Mesoporous 3Dhexagonal organosilicate films: Post-synthesis grafting vs. direct synthesis. Thin Solid Films 2006, 495, 175-179.

(35) Yuan, Y.; Chen, Y.; Chen, W.; Hong, R. Preparation, durability and thermostability of hydrophobic antireflective coatings for solar glass covers. Sol. Energy 2015, 118, 222-231.

(36) Franceschini, E. A.; de la Llave, E.; Williams, F. J.; Soler-Illia, G. J. A. A. A simple three step method for selective placement of organic groups in mesoporous silica thin films. Mater. Chem. Phys. 2015, 169, $82-88$.

(37) Sun, Y.; Zheng, J.; Huang, R.; Zhang, X.; Chen, C.; Jiang, B.; Chen, H.; Yan, L.; Yang, W. A simple method to control the microstructure and properties of sol-gel silica antireflective coatings. RSC Adv. 2017, 7, 31950-31959.

(38) von Graberg, T.; Hartmann, P.; Rein, A.; Gross, S.; Seelandt, B.; Röger, C.; Zieba, R.; Traut, A.; Wark, M.; Janek, J.; Smarsly, B. M. Mesoporous tin-doped indium oxide thin films: effect of mesostructure on electrical conductivity. Sci. Technol. Adv. Mater. 2011, 12, 025005 .

(39) Garcia, C.; Zhang, Y.; DiSalvo, F.; Wiesner, U. Mesoporous aluminosilicate materials with superparamagnetic $\mathrm{Fe}_{2} \mathrm{O}_{3}$ particles embedded in the walls. Angew. Chem., Int. Ed. 2003, 42, 1526-1530.

(40) Baklanov, M. R.; Mogilnikov, K. P.; Polovinkin, V. G.; Dultsev, F. N. Determination of pore size distribution in thin films by ellipsometric porosimetry. J. Vac. Sci. Technol., B: Microelectron. Nanometer Struct. 2000, 18, 1385-1391.

(41) Sing, K. S. W. Reporting physisorption data for gas/solid systems with special reference to the determination of surface area and porosity. Pure Appl. Chem. 1985, 57, 603-619. 
(42) Grosman, A.; Ortega, C. Capillary condensation in porous materials. Hysteresis and interaction mechanism without pore blocking / percolation process. Langmuir 2008, 24, 3977-3986.

(43) Etienne, M.; Quach, A.; Grosso, D.; Nicole, L.; Sanchez, C.; Walcarius, A. Molecular transport into mesostructured silica thin films: Electrochemical monitoring and comparison between p6m, P63/mmc, and Pm3n structures. Chem. Mater. 2007, 19, 844-856.

(44) Lide, D.; Milne, G. Handbook of Data on Organic Compounds, 3rd ed.; CRC Press, 1994; Vol. I, p 4951.

(45) Sindorf, D. W.; Maciel, G. E. Solid-state NMR studies of the reactions of silica surfaces with polyfunctional chloromethylsilanes and ethoxymethylsilanes. J. Am. Chem. Soc. 1983, 105, 3767-3776.

(46) Li, T.; He, J. A facile hybrid approach to high-performance broadband antireflective thin films with humidity resistance as well as mechanical robustness. J. Mater. Chem. C 2016, 4, 5342-5348.

(47) Inagaki, S.; Fukushima, Y. Adsorption of water vapor and hydrophobicity of ordered mesoporous silica, FSM-16. Microporous Mesoporous Mater. 1998, 21, 667-672.

(48) Boissiere, C.; Grosso, D.; Lepoutre, S.; Nicole, L.; Bruneau, A. B.; Sanchez, C. Porosity and mechanical properties of mesoporous thin films assessed by environmental ellipsometric porosimetry. Langmuir 2005, 21, 12362-12371.

(49) Juan-Díaz, M. J.; Martínez-Ibáñez, M.; Hernández-Escolano, M.; Cabedo, L.; Izquierdo, R.; Suay, J.; Gurruchaga, M.; Goñi, I. Study of the degradation of hybrid sol-gel coatings in aqueous medium. Prog. Org. Coat. 2014, 77, 1799-1806.

(50) Nielsen, K. H.; Kittel, T.; Wondraczek, K.; Wondraczek, L. Optical breathing of nano-porous antireflective coatings through adsorption and desorption of water. Sci. Rep. 2014, 4, No. 6595.

(51) Ye, T.; Wynn, D.; Dudek, R.; Borguet, E. Photoreactivity of alkylsiloxane self-assembled monolayers on silicon oxide surfaces. Langmuir 2001, 17, 4497-4500.

(52) Ye, T.; McArthur, E. A.; Borguet, E. Mechanism of UV photoreactivity of alkylsiloxane self-assembled monolayers. J. Phys. Chem. B 2005, 109, 9927-9938.

(53) Sanchez, C.; Belleville, P.; Popall, M.; Nicole, L. Applications of advanced hybrid organic-inorganic nanomaterials: From laboratory to market. Chem. Soc. Rev. 2011, 40, 696.

(54) Innocenzi, P.; Malfatti, L. Mesoporous thin films: Properties and applications. Chem. Soc. Rev. 2013, 42, No. 4198. 